\title{
INTERACTION OF ARTEMISININ AND TETRACYCLINE OR ERYTHROMYCIN AGAINST PLASMODIUM FALCIPARUM IN VITRO
}

\author{
YE* Z. and VAN DYKE K.**
}

\section{Summary :}

Antimalarial activities of tetracycline (TC) and erythromycin (EM), alone or in combination with artemisinin (Qinghaosu, QHS), were studied using chloroquine (CQ)-sensitive (D6) and -resistant (W2) strains of Plasmodium falciparum in vitro. The antimalarial potency of TC $\| C_{50}=9862 \mathrm{nM}$ for the $C Q$-sensitive parasite, 32414 $\mathrm{nM}$ for the $\mathrm{CQ}$-resistant one) or $\mathrm{EM}(\mathrm{IC} 50=45787 \mathrm{nM}$ for the $C Q$-sensitive parasite, $33397 \mathrm{nM}$ for the $C Q$-resistant one) was much less than that of $Q H S$ (IC 50 ranging from 25 to $40 \mathrm{nM})$. The CQ-resistant falciparum parasite displayed a cross-resistance to TC, while both the drug-sensitive and -resistant parasites exhibited similar responses to EM. However, antimalarial potency of EM appeared to be less than that of TC against the drug-sensitive parasite. When TC was combined with QHS, an additive interaction was observed against the $C Q$-sensitive falciparum parasite, while synergism was found with the CQ-resistant parasite. When EM was tested in combination with QHS, a potentiating interaction occurred with both the CQ-sensitive and -resistant falciparum parasite. The above results indicated that the QHS combination with either TC or EM may be a promising antimalarial preparation with low recrudescence compared to artemisinin used alone in clinical practice.

KEY WORDS : artemisinin. tetracycline. erythromycin. Plasmodium falciparum. antimalarials. antibiotics. drug combination.

\section{INTRODUCTION}

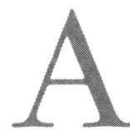

rtemisinin (Qinghaosu, QHS), isolated from a Chinese medicinal herb, Artemisia annua, is a new antimalarial drug characterized by a rapid onset of therapeutic effects against this infection (Klayman, 1985). Since chloroquine (CQ) resistant falciparum malaria has spread rapidly world-wide, QHS presents a serious challenge to the commonly-used drug chloroquine (Jiang et al., 1982; Anon 1982a). Three derivatives of QHS, artemether, artesunate and arteether are currently undergoing clinical trials. In spite of their potency and low toxicity, recrudescerice (return of the infection) is intolerably high in patients

*Department of Pharmacology, Institute of Chinese Materia Medica, China Academy of Traditional Chinese Medicine. Beijing 100700, China

***Department of Pharmacology and Toxicology, Robert C. Byrd Health Sciences Center of West Virginia University, Morgantown, WV 26506, U. S

Corresponding author: Knox Van Dyke, Tel: (304)-293-5449, Fax: (304)-293-6854
Résumé : INTERACTION DE L'ARTÉMISININE ET DE LA TETRACYCLINE OU L'ÉRYTHROMYCIN SUR PLASMODIUM FALCIPARUM IN VTTRO

Les activités antimalariques de la tétracycline (TC) et de l'érythromycine (EM) seules ou associées avec l'artémisinine (Qinghaosu, QHS) ont été évaluées in vitro sur deux souches de Plasmodium falciparum, une souche chloroquino-sensible (CQ-S) D6, et une chloroquino-résistante (CQ-R), la souche W2. Les propriétés antimalariques observées avec TC $\| C_{50}=9862 \mathrm{nM} C Q-S, 32414 \mathrm{nM}$ $C Q-R)$ ou EM (IC $50=45787$ nM CQ-S, 33397 nM CQ-R) respectivement sont beaucoup plus faibles que celles observées avec QHS $\| C_{50}=25$ à $\left.40 \mathrm{nM}\right)$. La souche CQ-R montre une résistance croisée avec TC alors que les deux souches ont la même sensibilité vis-à-vis de EM. Toutefois les propriétés antimalariques de EM sont plus faibles que celles de TC vis-à-vis de la souche CQ-S. Lorsque TC est associé avec QHS, un effet additif est observé vis-à-vis de la souche CQ-S; avec la souche CQ-R, il se produit une synergie. L'association EM plus QHS provoque une synergie sur les deux souches étudiées. Ces résultats montrent que l'artémisinine associée avec soit la tétracycline soit l'érythromycine pourraient se révéler être des associations antimalariques provoquant peu de rechutes en comparaison à ce qui est observé avec l'artémisinine lors de son utilisation clinique.

MOTS CLES : artémisinine, tétracycline. érythromycine. Plasmodium falciparum. antimalariques. antibiotiques, association thérapeutique.

receiving QHS or QHS-like drugs (Li et al., 1984). The recrudescence probably results from the short half-life of QHS and its derivatives in plasma (Anon, 1982b). Recently, in order to overcome the recrudescence, work has been directed towards studying a combination of QHS or its derivatives with other antimalarial compounds. Some combinations of QHS with antimalarial drugs, for instance, QHS + CQ, or QHS + pyrimethamine, antagonize each other (Chawira and Warhurst, 1987), while others are potentiating and found very promising. The potentiating action of QHS and mefloquine or tetracycline (TC) both in vitro and in vivo was reported (Chawira and Warhurst 1987; Chawira et al., 1987). Previously, we demonstrated a potentiation of QHS and bisbenzylisoquinolines such as tetrandrine or berbamine against falciparum parasite in vitro (Ye et al., 1989; Ye et al., 1993).

In this present study, we explored an interaction of QHS and erythromycin (EM), as well as QHS and TC against Plasmodium falciparum in vitro. Although the combination of EM and other antimalarial drugs have 
been extensively investigated (Khan et al., 1991; Gingras and Jensen 1993; Gingras and Jensen 1992), its combination with QHS, to our knowledge, has not yet been reported. Although a potentiating synergism in the combination of QHS and TC was shown using NF54 ( CQ-sensitive) and K1 (CQ-resistant) strains of falciparum parasite (Chawira and Warhurst, 1987), different responses among the various parasitic isolates to an antibiotics or its combination were reported (Khan et al., 1991; Gingras and Jensen, 1992; Gershon and Howells, 1984; Reacher et al., 1981; Phillips et al., 1984; Raichowdhuri and Gajanana, 1984). Therefore, West Africa strain (D6, a CQ-sensitive) and Indochina strain (W2, CQ-resistant) were used in our experiments to see if there are any difference between different strains of $P$. falciparum parasites in interaction between QHS and TC.

\section{MATERIALS AND METHODS}

\author{
CULTURES AND TESTS :
}

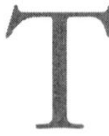
wo strains of Plasmodium falciparum were used in these experiments : one is the CQsensitive West Africa (D6) strain and the other is the CQ-resistant Indochina (W2) strain (Martin et al., 1987). Both of them, kindly donated by Dr. Robert Miller, Walter Reed Army Institute of Research, were cultured in the complete medium of RPMI 1640 according to the candle jar method (Jensen and Trager, 1977).

Artemisinin (QHS) is a gift from Dr D.L. Klayman of the Walter Reed Army Institute of Research. A stock solution of QHS (10-3 M) was prepared by dissolving it in dimethylsulfoxide (DMSO) because of its insolubility in water. Before adding to the culture, this stock solution was diluted with RPMI 1640 medium without serum to yield a solution with drug concentration of $10^{-4} \mathrm{M}$. After the drug solution was sterilized by filtering it using a $0.22 \mu \mathrm{M}$ cellulose acetate membrane, further serial dilutions of the drug were prepared in the complete medium of RPMI 1640 containing 10\% pooled human A+ serum, $25 \mathrm{mM}$ HEPES, and $25 \mathrm{mM}$ NaHCO3. Preliminary experiments showed that final DMSO concentrations of $0.2 \%$ or less had no detectable effect on parasite growth. TC hydrochloride and EM, purchased from Sigma Chemicals, were respectively dissolved in RPMI 1640 medium to produce $10^{-2} \mathrm{M}$ solution, and sterilized and diluted in a similar manner to QHS.

For a given experiment, 4-day-old Petri dish cultures with a $5-10 \%$ parasitemia were diluted with medium containing 25\% non-parasitized human erythrocyte type A+ to obtain a culture with a final hematocrit of $1.5 \%$ and a parasitemia of $0.5-1.0 \%$.
Antimalarial activity was assessed using the method of Desjardins and co-workers (1979), except that tritiated adenosine was substituted for tritiated hypoxanthine as a precursor for parasite nucleic acids (Ye et al., 1987). Briefly, the final volume $(250 \mu \mathrm{l})$ in each well of a 96-well microtiter plate consisted of : (1) $25 \mu \mathrm{l}$ of complete medium with or without drug or drug combination; and (2) $200 \mu \mathrm{l}$ of the parasitized cultures or non-parasitized human erythrocytes as a control. After incubation of the plates in a candle jar for 24 hours at $37^{\circ} \mathrm{C}, 25 \mu \mathrm{l}$ of $[2,8-3 \mathrm{H}]$ adenosine $(0.5 \mu \mathrm{Ci})$ with a specific activity of $59.20 \mathrm{Ci} / \mathrm{mMole}$ was added to each well. The plates were then incubated at $37^{\circ} \mathrm{C}$ for an additional 18 hours. At the end of the incubation, the contents in each well were harvested onto fiberglass filter disks using a Bellco semi-automated cell harvester. The filters were rinsed with distilled water six times and each disk was placed in a scintillation vial with $5.0 \mathrm{ml}$ Cytoscinttm liquid scintillation fluid. Radioactivity was counted using a Packard Tri-Carb Model 1900 CA scintillation spectrometer.

\section{DRUGS' INTERACTION :}

In order to examine any interaction between the two drugs, the compounds were mixed in fixed ratios as described by Martin and colleagues (1987). Three concentrations of TC or EM, i.e., $1 \times 10^{-4}, 2 \times 10^{-4}$ and $3 \times 10^{-4} \mathrm{M}$, were mixed with QHS at concentrations of $3 \times 10^{-7}, 2 \times 10^{-7}$ and $1 \times 10^{-7} \mathrm{M}$, respectively. Ratios of $\mathrm{TC}$ or $\mathrm{EM} / \mathrm{QHS}$ in concentration were, respectively, 1000:3, 1000:1 and 3000:1, which were designated as Combination 1, 2, and 3. A series of dilutions (continuous one to one dilution) were made respectively from Combination 1, 2, and 3. Antimalarial efficacies of the Combination 1,2, and 3, as well as QHS, TC, or EM alone were assessed using Desjardins' method. Based on inhibitory percentage-concentrations of the drug combinations, $\mathrm{IC}_{50}$ values of an individual drug in the Combination 1,2 and 3 were calculated using probit analysis. Sum of fractional inhibitory concentration (SFIC) of the drug combination was calculated based on its $\mathrm{IC}_{50}$ value (SFIC=IC 50 of one drug in the combination/IC50 of the corresponding drug alone $+\mathrm{IC}_{50}$ of the other drug in the combination/ $/ \mathrm{I}_{50}$ of the corresponding drug alone) and then isobolograms were constructed as described by Berenbaum (1978). Usually, the interaction of two drugs in combination exhibits three possibilities : antagonism, addition, and synergism. This is illustrated by the following example: $1+1<2$ (antagonism), $1+1=2$ (addition), and $1+1>2$ (potentiation or synergism).

Duplicate wells were prepared for each drug concentration and experiments were repeated a total of three times. 


\section{RESULTS}

A s compared to QHS, antimalarial potency of TC or EM is very low (See Table I and III). It seems that $\mathrm{CQ}$-resistant strain of $P$. falciparum exerts cross resistance to TC, because IC50 of TC for the resistant parasite is significantly higher $(\mathrm{p}<$ 0.05) than its $\mathrm{IC}_{50}$ for the sensitive one. However, responses of both the sensitive and resistant falciparum parasites to EM were similar, e.g., IC50 values: 45787 vs $33397 \mathrm{nM}$ (See Table III). EM is less potent than TC in antimalarial activity against the drug sensitive parasite based on comparison of their IC50 values: 45787 vs $9862 \mathrm{nM}$ (See Tables I and III), while, with the CQresistant parasite, the two antibiotics appeared to be similarly potent (See Tables I and III).

When TC or EM was combined with QHS, IC50 values of QHS in the combination 1, 2, and 3 are significantly lower than that of QHS alone, regardless of which strain of falciparum parasite was used. And decrease of QHS's IC50 value was observed with increase of TC or EM dose in the combination. An additive interaction between TC and QHS was found in the drug sensitive parasite because SFIC values in the combination of TC and QHS are close to 1.0, while a synergistic action in the combination occurred in the drug resistant parasite with SFIC values significantly less than 1.0 (See Table II), which was confirmed by isobolograms in Fig. 1, namely, the isobologram of the drug combination against the CQsensitive parasite is very close to the control line (a diagonal line in Fig. 1), displaying addition, and the isobologram for the CQ-resistance is concave, indicating synergism.

However, when EM was combined with QHS, interaction between EM and QHS against both the sensitive and the resistant strains of falciparum parasite was observed to be synergestic. As shown in Table IV, all the SFIC values in the combination of EM and QHS are less than 1.0. In addition, the shape of isobolograms for both strains of falciparum parasites in Fig. 2 are concave, indicating the potentiating interaction occurred in the combination.

\section{DISCUSSION}

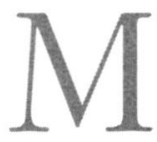

any studies on antimalarial activity of antibiotics, especially TC and EM, have been done, but neither TC nor EM alone can be developed into a single antimalarial drug because of its weak efficacy and slow onset of antimalarial action. Our data in this study also demonstrated that TC or EM alone cannot be used as a single antimala- rial drug because their antimalarial potency is too low as compared to that of QHS. There is from several hundred to over one thousand fold difference between TC or EM and QHS in IC50 values. Probably, their slow and weak antimalarial action may result from their slow uptake and low accumulation in the parasitized red cells (Geary et al., 1988). Therefore, it is unlikely that TC or EM itself could be developed into a single antimalarial drug.

However, a combination of TC or EM and other antimalarials is a practical way to develop a new antimalarial preparation for treating and preventing the CQ-resistant falciparum malaria because the antibiotics were not only able to increase antimalarial efficacy of other drugs (Gingras and Jensen, 1993; Gershon and Howells, 1984; Gershon and Howells, 1986; Noeypatimanond et al., 1983; Watt et al., 1992), but also delay emergence of resistance of falciparum parasite to an individual drug in the combination (Puri and Dutta, 1989). Owing to these advantages of the combination of the antibiotics and other antimalarials, various combinations of TC or EM with other antimalarial drugs such as quinine, CQ, and amodiaquine have been investigated. Up to now, the combination of quinine and TC is a standard regimen for treatment of the resistant falciparum malaria (Watt et al., 1992; Looareesuwan et al., 1992). However, attenuation of the parasite sensitivity to the combination of quinine and TC evoked development of a combination of EM and other antimalarials (Looareesuwan et al., 1992). In addition, TC cannot be used for both children under 8 and pregnant women (White, 1983). Therefore, there is an urgent need for an alternative combination like EM and other antimalarials. EM was shown to have antimalarial activity in vitro ( $P$. falciparum) (McColm and McHardy, 1984; Geary and Jensen, 1983) and in vivo (P.berghei and P. knowlesi) (Warhurst et al., 1976; Warhust et al., 1983) and a potentiating synergism was manifested in its combination with CQ (Gershon and Howells, 1984; Warhurst et al., 1976). However, the combination of EM and CQ or quinine failed to treat falciparum malaria in clinical trials (Phillips et al., 1984; Pang et al., 1985; Brandling-Bennett et al., 1988). It seems that the combination of EM with 4-aminoquinolines should not be developed into an antimalarial preparation based on current data available.

Owing to the failure of the EM combination with 4aminoquinolines in clinical trials and the attenuation of the TC combination with quinine to the CQ-resistant falciparum malaria, a combination of QHS or its derivatives such as artemether and artesunate with other antimalarials including antibiotics have been explored (Jiang et al., 1982; Li et al., 1984; Shwe et 


\begin{tabular}{ccccc}
\hline \multicolumn{4}{c}{ Single Drug } & \multicolumn{3}{c}{ Drug Combinationb } \\
\hline TC & QHS & TC $(300 \mu \mathrm{M})$ & TC $(200 \mu \mathrm{M})$ & TC $(100 \mu \mathrm{M})$ \\
$(1-100 \mu \mathrm{M})$ & $(0.001-0.1 \mu \mathrm{M})$ & $\mathrm{Q} \mathrm{HS}(0.1 \mu \mathrm{M})$ & $\mathrm{QHS}(0.2 \mu \mathrm{M})$ & $\mathrm{QHS}(0.3 \mu \mathrm{M})$ \\
\hline $\mathrm{S} 9862 \pm 3777$ & $37.3 \pm 8.7$ & $9441 \pm 456(\mathrm{TC})$ & $6996 \pm 2669(\mathrm{TC})$ & $4609 \pm 542(\mathrm{TC})$ \\
& & $3.2 \pm 1.5(\mathrm{QHS})$ & $7.0 \pm 2.7(\mathrm{QHS})$ & $13.9 \pm 1.6(\mathrm{QHS})$ \\
$\mathrm{R} \mathrm{32414 \pm 4017}$ & \multirow{4}{*}{$40.2 \pm 8.2$} & $14754 \pm 5162(\mathrm{TC})$ & $12378 \pm 4261(\mathrm{TC})$ & $5659 \pm 3123(\mathrm{TC})$ \\
& & $4.9 \pm 1.7(\mathrm{QHS})$ & $12.4 \pm 4.3(\mathrm{QHS})$ & $17.0 \pm 9.4(\mathrm{QHS})$
\end{tabular}

Table I. - $\mathrm{IC}_{50}(\mathrm{nM})$ of Tetracycline and Artemisinin for Each Drug Alone and in Combination ${ }^{\mathrm{a}}$ a. The data in the table above are the mean values \pm S.D. (nM) from three experiments; TC and QHS represent tetracycline and artemisinin, respectively; $\mathrm{S}$ and $\mathrm{R}$ represent CQ-sensitive and resistant strains of $P$. falciparum. b. Ratios of TC/QHS in the drug combinations are 3000;1, 1000:1, and 1000:3, respectively.

\begin{tabular}{|c|c|c|c|}
\hline \multirow[t]{3}{*}{ Parasiteb } & \multicolumn{3}{|c|}{ SFICC } \\
\hline & $300 \mu \mathrm{M}(\mathrm{TC})$ & $200 \mu \mathrm{M}(\mathrm{TC})$ & $100 \mu \mathrm{M}(\mathrm{TC})$ \\
\hline & $0.1 \mu \mathrm{M}(\mathrm{QHS})$ & $0.2 \mu \mathrm{M}(\mathrm{QHS})$ & $0.3 \mu \mathrm{M}(\mathrm{QHS})$ \\
\hline S strain & $1.03 \pm 0.18$ & $0.92 \pm 0.15$ & $0.90 \pm 0.16$ \\
\hline $\mathrm{R}$ strain & $0.57 \pm 0.15$ & $0.69 \pm 0.19$ & $0.60 \pm 0.22$ \\
\hline \multicolumn{4}{|c|}{$\begin{array}{l}\text { Table II. - Effect of Combination of Tetracycline and Artemisinin on P. falcipa- } \\
\text { rum }^{\text {a }}\end{array}$} \\
\hline \multicolumn{4}{|c|}{$\begin{array}{l}\text { a. The data in the table above are the mean values } \pm \text { S.D from three experiments. } \\
\text { b. } S \text { and } \mathrm{R} \text { strains represent } \mathrm{CQ} \text {-sensitive and resistant strains of } P \text {. falciparum. } \\
\text { c. SFIC represents sum of fractional inhibitory concentration. SFIC is equal to one in cases } \\
\text { of additive effects of the drugs, higher than one in cases of antagonism, and lower than } \\
\text { one in synergistic action. }\end{array}$} \\
\hline
\end{tabular}

\begin{tabular}{cccccc}
\hline & \multicolumn{2}{c}{ Single Drug } & \multicolumn{3}{c}{ Drug Combination $^{\text {b }}$} \\
\cline { 2 - 6 } & EM & QHS & EM $(300 \mu \mathrm{M})$ & EM $(200 \mu \mathrm{M})$ & EM $(100 \mu \mathrm{M})$ \\
& $(1-100 \mu \mathrm{M})$ & $(0.001-0.1 \mu \mathrm{M})$ & QHS $(0.1 \mu \mathrm{M})$ & QHS $(0.2 \mu \mathrm{M})$ & QHS $(0.3 \mu \mathrm{M})$ \\
\hline $\mathrm{S}$ & $45787 \pm 28248$ & $25.1 \pm 7.2$ & $17160 \pm 8234(\mathrm{EM})$ & $12164 \pm 6092(\mathrm{EM})$ & $4955 \pm 3907(\mathrm{EM})$ \\
& & $5.7 \pm 2.7(\mathrm{QHS})$ & $12.2 \pm 4.1(\mathrm{QHS})$ & $14.9 \pm 1.2(\mathrm{QHS})$ \\
& & & & \\
$\mathrm{R}$ & \multirow{3}{*}{$26.2 \pm 1.1$} & $17163 \pm 718(\mathrm{EM})$ & $12165 \pm 5065(\mathrm{EM})$ & $4616 \pm 675(\mathrm{EM})$ \\
& $33397 \pm 9639$ & & $5.7 \pm 0.2(\mathrm{QHS})$ & $12.2 \pm 5.1(\mathrm{QHS})$ & $13.9 \pm 2.0(\mathrm{QHS})$
\end{tabular}

Table III. - IC 50 (nM) of Erythromycin and Artemisinin for Each Drug Alone and in Combination ${ }^{\mathrm{a}}$

a. The data in the table above are the mean values \pm S.D. (nM) from three experiments; EM and QHS represent erythromycin and artemisinin, respectively; $S$ and $R$ represent CQ-sensitive and resistant strain of $P$. falciparum.

b. Ratios of EM/QHS in the drug combinations are 3000:1, 1000:1, and 1000:3, respectively.

\begin{tabular}{llll}
\hline Parasiteb & \multicolumn{3}{c}{ SFICC } \\
\cline { 2 - 4 } & $300 \mu \mathrm{M}(\mathrm{EM})$ & $200 \mu \mathrm{M}(\mathrm{EM})$ & $100 \mu \mathrm{M}(\mathrm{EM})$ \\
& $0.1 \mu \mathrm{M}(\mathrm{QHS})$ & $0.2 \mu \mathrm{M}(\mathrm{QHS})$ & $0.3 \mu \mathrm{M}(\mathrm{QHS})$ \\
\hline S strain & $0.69 \pm 0.17$ & $0.82 \pm 0.10$ & $0.69 \pm 0.16$ \\
\multirow{2}{*}{ R strain } & $0.76 \pm 0.11$ & $0.83 \pm 0.09$ & $0.67 \pm 0.10$
\end{tabular}

Table IV. - Effect of Combination of Erythromycin and Artemisinin on P. falciparum ${ }^{\mathrm{a}}$.

a. The data in the table above are the mean values \pm S.D from three experiments. EM and QHS represent erythromycin and artemisinin respectively.

b. S and R strains represent CQ-sensitive and resistant strains of P. falciparum.

c. SFIC represents sum of fractional inhibitory concentration. SFIC is equal to one in cases

of additive effects of the drugs, higher than one in cases of antagonism, and lower than

one in synergistic action. 
al., 1988; Shwe et al., 1989; Win et al., 1992; Looareesuwan et al., 1992). Although QHS is a new antimalarial with rapid onset and relatively low toxicity, its clinical application has been limited because of its high recrudescence, probably due to short half life in the human. In order to circumvent its recrudescence, a combination of QHS with other antimalarial drugs may be a practical solution. Some experiments in vitro and in vivo in laboratories have demonstrated that the combinations of QHS with other antimalarial drugs such as mefloquine, tetrandrine, berbamine, and TC may be promising candidates for new antimalarial preparations which may attenuate recrudescence of QHS. Actually, the combinations of QHS and mefloquine or QHS and TC significantly decrease the recrudescence as compared to QHS used alone in clinical trials (Jiang et al., 1982; Li et al., 1984; Sy et al., 1993). Probably, this is because potentiating interaction in those combinations can enhance antimalarial efficacy of QHS so much that a residual parasite could not escape being attacked by QHS, and the drugs above mentioned in the combination with QHS have a relatively longer half-life in the human, which may compensate for short half-life of QHS causing the recrudescence.

Using D6 and w2 strains of falciparum parasite, synergistic or additive interaction occurred in the present study when QHS was combined with TC (See Table II and Fig. 1). The data obtained from these experiments confirmed therapeutic effectiveness of the combination of QHS and TC as a new antimalarial regimen for treatment of patients infected with either the CQ-sensitive or resistant falciparum parasite. Although QHS exhibits relatively low toxicity, it can produce bone marrow depression or cardiovascular adverse effects (Anon, 1982c). Another advantage of the drug combination is to be able to decrease the dose of QHS, which itself would lead to less side effects. As shown in Table I, the dose of QHS in combination with TC is significantly lower than that of QHS alone based on a comparison of their IC 50 values. Therefore, the combination of QHS with TC can not only increase total antimalarial efficacy so that the recrudescence may be overcome, but also lead to possible lower toxicity of QHS. Therefore, it is necessary to conduct in various worldwide locations clinical trials on the QHS combination with TC on a large scale in order to evaluate the therapeutic importance of the combination.

In these experiments, we also revealed potentiating synergism in the combination of EM and QHS against both the drug sensitive and resistant strains of parasite, indicating EM might be useful in combination with QHS as an alternative antimalarial preparation, since QHS is quite different from 4-aminoquinolines in chemical structure and in antimalarial mechanism (Klayman, 1985; Ye et al., 1983; Lee et al., 1988). Unlike TC, the drug resistant parasite in our experiments did not possess a cross resistance to EM, although the antimalarial potency of EM against the drug sensitive parasite is less than TC. However, some data obtained from another laboratory demonstrated higher antimalarial potency of EM than TC (McColm and McHardy, 1984). In addition, EM has properties of being inexpensive, readily available, and not having deleterious effects during odontogenesis, so that it can be used safely in the treatment of pregnant women and young children. Therefore, this combination warrants clinical trial as well as a further investigation using different isolates of falciparum parasite.

Mechanistic experiments revealed that TC and EM exert their antimalarial effects by inhibiting protein synthesis in microorganisms, and especially, they are potent inhibitors on protein synthesis in mitochondrion (Blum et al., 1984; Divo et al., 1985; Prapunwattana et al., 1988; Kiatfuengfoo et al., 1989). Similarly, QHS was found to have remarkable attack on mitochondrion in the parasite (Jiang et al., 1985). Also, protein synthesis in the parasite was proven to be probably the earliest and main target of antimalarial action by QHS (Gu et al., 1983). Usually, synergism occurs when the two drugs in a combination attack different chain of the same biochemical pathway. Presumably, because of possible similarity in mechanism by which TC or EM, and QHS exert their antimalarial activity, synergistic effects were found in the combination of TC or EM and QHS against the CQ-resistant falciparum parasite and in the combination of EM and QHS against sensitive one. However, we cannot explain why the combination of TC and QHS was found to be an additive interaction against the CQ-sensitive parasite. Usually, an additive interaction manifests itself when the targets of the two drugs in a combination are independent of each other in biochemical pathways. If this is true, it is implied that some biochemical pathways, especially those related to protein synthesis in mitochondria, have been changed when the parasite is developed into the resistant one.

Owing to the fact that the CQ-resistant strain of $P$. falciparum exhibits a cross-resistance to TC in this investigation, this makes it more difficult to explain the reason why the drug resistant parasite is refractory to respond to $\mathrm{TC}$, but the combination of $\mathrm{TC}$ and QHS possess synergism against the drug resistant parasite. However, a study using some isolates of falciparum parasite other than D6 or W2 strain revealed that TC had similar antimalarial potency against both the sensitive and the resistant strains of $P$. falciparum 


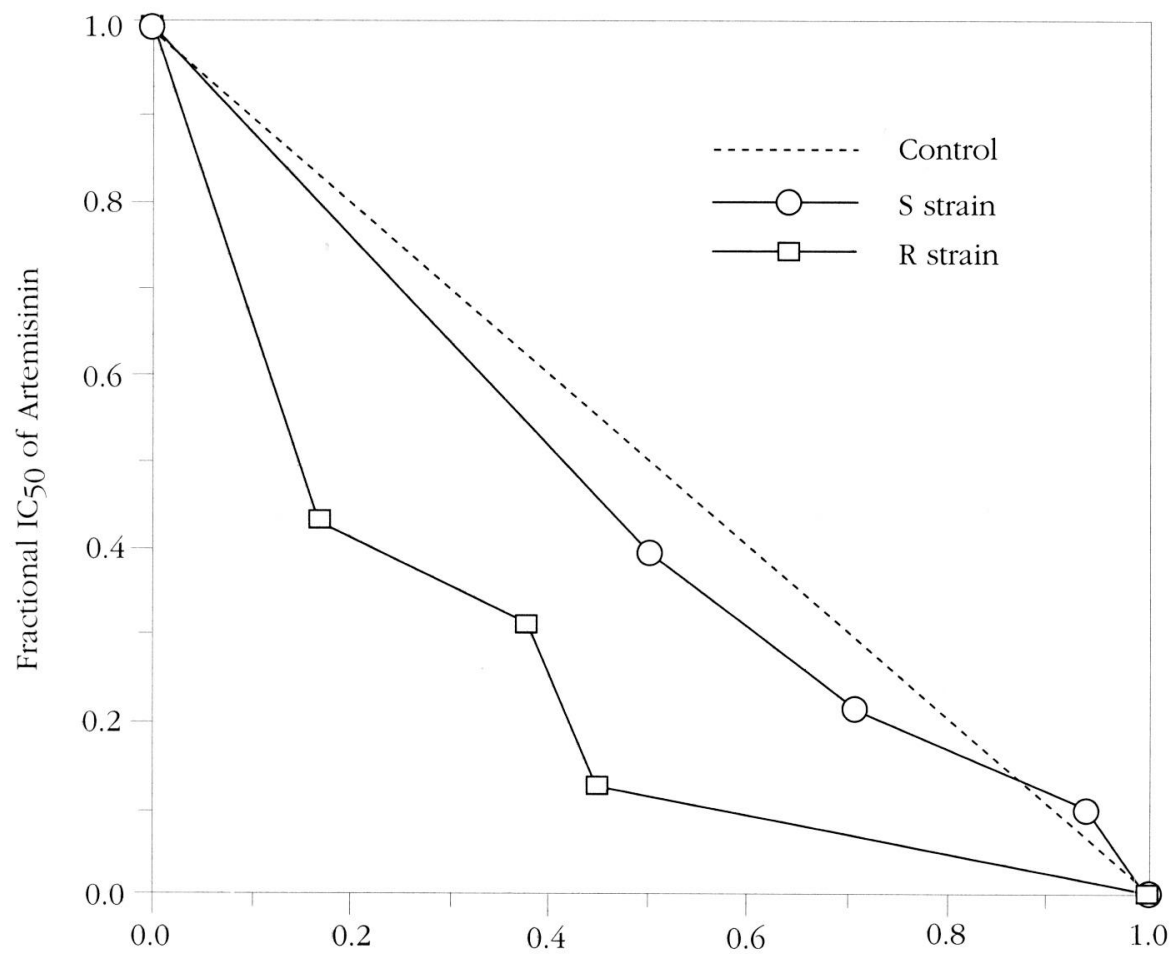

Fractional $\mathrm{IC}_{50}$ of Tetracycline

Fig. 1 - Isobolograms of interaction between artemisinin and tetracycline against CQ-sensitive (S) and CQ-resistant (R) strains of Plasmodium faciparum in vitro.

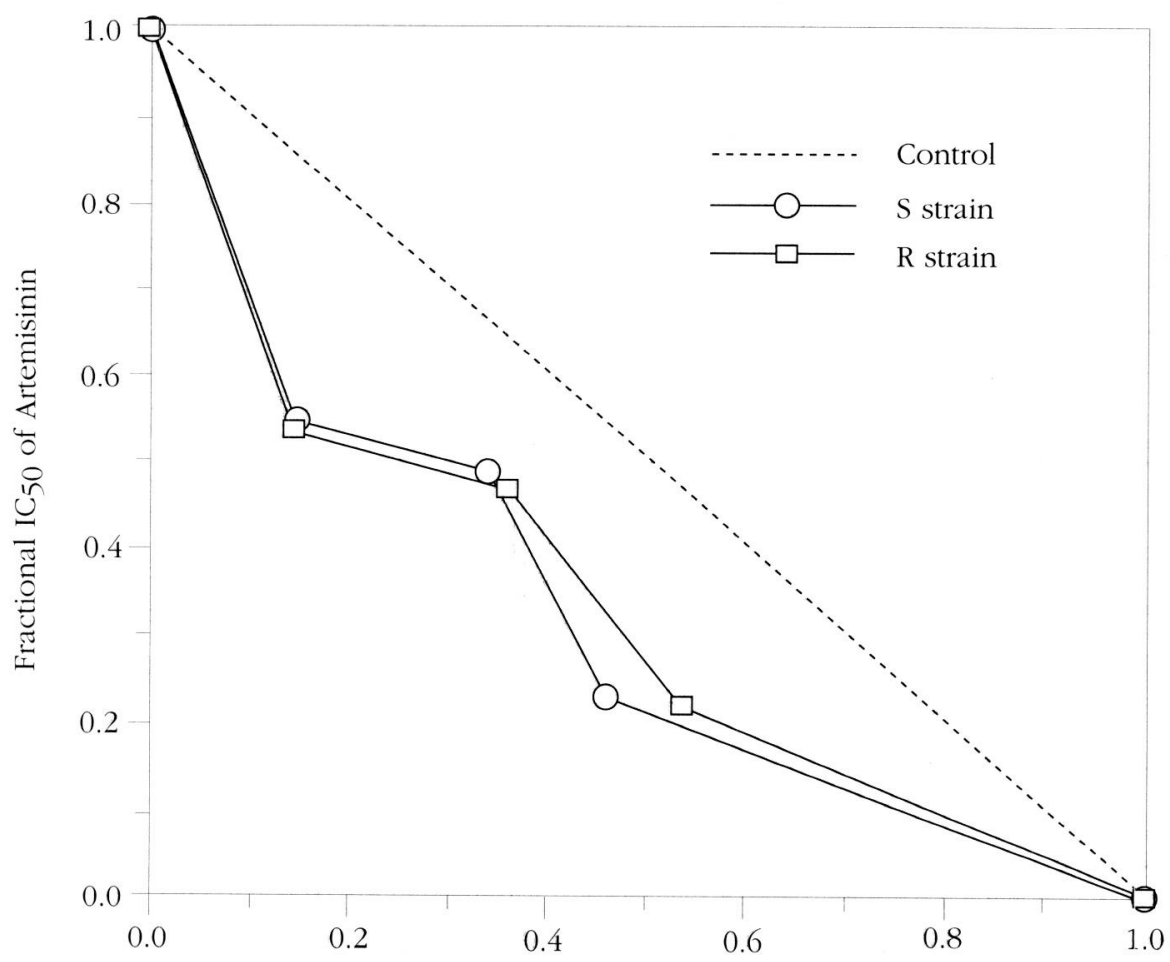

Fractional IC50 of Erythromycin

Fig. 2 - Isobolograms of interaction between artemisinin and erythromycin against CQ-sensitive (S) and CQ-resistant (R) strains of Plasmodium faciparum in vitro 
(Chawira and Warhurst, 1987). The fact that various isolates of the drug-resistant falciparum parasite have different responses to an individual drug leads to a consideration that the mechanism by which different isolates of the parasite are developed into the resistance to CQ is somehow different.

In sum, the QHS combination with $\mathrm{TC}$ is a promising candidate as an alternative antimalarial preparation for treatment of malarial infections, especially the CQresistant falciparum malaria. Also, the QHS combination with EM needs further investigation both in laboratories and in clinical trials.

\section{REFERENCES}

ANON : Clinical studies on the treatment of malaria with qinghaosu and its derivatives as antimalarials. Journal of Traditional Chinese Medicine, 1982a, 2, 45-50

ANON : Metabolism and pharmakinetics of qinghaosu and its derivatives. Journal of Traditional Chinese Medicine, 1982b, 2, 25-30.

ANon: Studies on the toxicity of qinghaosu and its derivatives. Journal of Traditional Chinese Medicine, 1982c, 2, 31-38.

Berenbaum M.C. : A method for testing for synergy with any number of agents. Journal of Infectious Diseases, 1978, 137, 122-130.

Blum J.J., Yayon A., Friedman S. and Ginsburg H. : Effects of mitochondrial protein synthesis inhibitors on the incorporation of isoleucine into Plasmodium falciparum in vitro. Journal of Protozoology, 1984, 31, 475-479.

Brandling-Bennett A.D., Oloo A.J., Khan B. and Watkins W. M. : Failure of erythromycin to improve chloroquine treatment of Plasmodium falciparum malaria in Kenya. Transactions of The Royal Society of Tropical Medicine and Hygiene, 1988, 82, 363-365.

Chawira A.N. and Warhurst D.C. : The effect of artemisinin combined with standard antimalarials against chloroquine-sensitive and chloroquine-resistant strains of Plasmodium falciparum in vitro. Journal of Tropical Medicine and Hygiene, 1987, 90, 1-8.

Chawira A.N., Warhurst D. C., Robinson B. L. and Peters W. : The effect of combination of qinghaosu (artemisinin) with standard antimalarial drugs in the suppressive treatment of malaria in mice. Transactions of The Royal Society of Tropical Medicine and Hygiene, 1987, 81, 554558.

Desjardins R.E., Canfield C.J., Haynes J.D. and Chulay J.D. : Quantitative assessment of antimalarial activity in vitro by a semiautomated microdilution technique. Antimicrobial Agents and Chemotherapy, 1979, 16, 710-718.

Divo A.A., Geary T.G. and Jensen J.B. : Oxygen- and timedependent effects of antibiotics and selected mitochondrial inhibitors on Plasmodium falciparum in culture. Antimicrobial Agents and Chemotherapy, 1985, 27, 21-27.
Geary T.G., Divo A.A. and Jensen J.B. : Uptake of antibiotics by Plasmodium falciparum in culture. American Journal of Tropical Medicine and Hygiene, 1988, 38, 466-469.

Geary T.G. and Jensen J.B. : Effects of antibiotics on Plasmodium falciparum in vitro. American Journal of Tropical Medicine and Hygiene, 1983, 32, 221-225.

Gershon P. and Howells R.E. : Combination of the antibiotics erythromycin and tetracycline with three standard antimalarials against Plasmodium falciparum in vitro. Annals of Tropical Medicine and Parasitology, 1984, 78, $1-11$.

Gershon P. and Howells R.E. : Synergy of four macrolide antibiotics with chloroquine against chloroquine-resistant Plasmodium falciparum in vitro. Transactions of the Royal Society of Tropical Medicine and Hygiene, 1986, 80, 753-757.

Gingras B. and Jensen J.B. : Activity of azithromycin (CP62,993) and erythromycin against chloroquine-sensitive and chloroquine-resistant strains of Plasmodium falciparum in vitro. American Journal of Tropical Medicine and Hygiene, 1992, 47, 378-382.

Gingras B.A. and JENSEN J.B. : Antimalarial activity of azithromycin and erythromycin against Plasmodium berghei. American Journal of Tropical Medicine and Hygiene, 1993, 49, 101-105.

Gu H., Warhust D.C. and Peters W. : Rapid action of qinghaosu and related drugs on incorporation of $[3 \mathrm{H}]$ isoleucine by Plasmodium falciparum in vitro. Biochemical Pharmacology, 1983, 32, 2463-2466.

JENSEN J.B. and Trager W. : Plasmodium falciparum in culture: Use of outdated erythrocytes and description of the candle jar method. Journal of Parasitology, 1977, 63, 883886.

Jiang J., Jacobs G., Liang D. and Aikawa M. : Qinghaosuinduced change in the morphology of Plasmodium inui. American Journal of Tropical Medicine and Hygiene, 1985, 34, 424-428.

Jiang J., Li G., Guo X. and Kong Y. : Antimalarial activity of mefloquine and qinghaosu. Lancet, 1982, 2, 285-288.

Khan B., Brandling-Bennett A.D., Watkins W.M., Oloo A.J., Ojoo P. and Koech D.K. : Plasmodium falciparum sensitivity to erythromycin and 4-aminoquinoline combinations in vitro. Annals of Tropical Medicine and Parasitology, 1991, 85, 215-222.

Kiatfuengfoo R., Suthiphongchai T., Prapunwattana P. and Yuthavong Y. : Mitochondria as the site of action of tetracycline on Plasmodium falciparum. Molecular and Biochemical Parasitology, 1989, 34, 109-116.

Klayman D.L. : Qinghaosu (artemisinin) : An antimalarial drug from China. Science, 1985, 228, 1049-1055

LeE P., Ye Z., VAn Dyke K. and KIRK R.G. : X-ray microanalysis of Plasmodium falciparum and infected red blood cells : Effects of qinghaosu and chloroquine on potassium, sodium, and phosphours composition. American Journal of Tropical Medicine and Hygiene, 1988, 39, 157165.

Li G., Arnold K., Guo X. and Jian H. : Randomised compa- 
rative study of mefloquine, qinghaosu, and pyrimethamine-sulfadoxine in patients with falciparum malaria. Lancet, 1984, 2, 1360-1361.

Looareesuwan S., Kyle D.E., Viravan C., Vnijanonta S., Wilairatana P., Charoenlarp P., Canfield C.J. and WebSTER H.K. : Treatment of patients with recrudescent falciparum malaria with a sequential combination of artesunate and mefloquine. American Journal of Tropical Medicine and Hygiene, 1992, 47, 794-799.

Looareesuwan S., Wilairatana P., Vanijanonta S., Kyle D. and Webster K. : Efficacy of quinine-tetracycline for acute uncomplicated falciparum malaria in Thailand. Lancet, 1992, 339, 369.

Martin S.K., Oduola A.M.J. and Milhous W.K. : Reversal of chloroquine resistance in Plasmodium falciparum by verapamil. Science, 1987, 235, 899-901.

McColm A.A. and McHardy N. : Evaluation of a range of antimicrobial agents against the parasitic protozoa, Plasmodium falciparum, Babesia rodhaini and Theileria parva in vitro. Annals of Tropical Medicine and Parasitology, 1984, 78, 345-354.

Noeypatimanond S., Malkul S., Benjapong W., Duriyananda D. and UngKasRithongkul M. : Treatment of Plasmodium falciparum malaria with a combination of amodiaquine and tetracycline in Central Thailand. Transactions of The Royal Society of Tropical Medicine and Hygiene, 1983, 77, 338-340.

Pang L.W., Boudreau E.F., Childs G.E., Webster H.K., Supanantalerk C. and Somutsakorn P. : Failure of largedose erythromycin in combination with a standard dose of chloroquine or quinine in the treatment of human falciparum malaria. Bulletin of the World Health Organization, 1985, 63, 739-743.

Phillips R.E., Looareesuwan S., Karbwang J., Warrell D.A., White N.J., Kasemsarn P. and Warhurst D.C. : Failure of chloroquine-erythromycin and chloroquine-tetracycline combinations in treatment of chloroquine-resistant falciparum malaria in Eastern Thailand. Lancet, 1984, 1, 300302.

Prapunwattana P., O'Sullivan W.J. and Juthavong Y. : Depression of Plasmodium falciparum dihydroorotate dehydrogenase activity in vitro culture by tetracycline. Molecular and Biochemical Parasitology, 1988, 27, 119124.

PURI S.K. and DutTa G.P. : Delay in emergence of mefloquine resistance in Plasmodium berghei by use of drug combinations. Acta Tropica, 1989, 46, 209-212.

Raichowdhuri A.N. and Gajanana A. : Antibiotic sensitivity of Plasmodium falciparum in vitro. Indian Journal of Medical Research, 1984, 80, 103-106.

Reacher M., Campbell C.C., Freeman J., Doberstyn E.B. and Brandling-Bennett A.D. : Drug therapy for Plasmodium falciparum malaria resistant to pyrimethamine sulfadoxine (Fansidar). Lancet, 1981, 2, 1066-1068.

Shwe T., Myint P.T., Myint W., Htut Y., Soe L. and Thwe M. : Clinical studies on treatment of cerebral malaria with artemether and mefloquine. Transactions of The Royal Society of Tropical Medicine and Hygiene, 1989, 83, 489.
Shwe T., Myint P. T., Htut Y., Myint W. and Soe L. : The effect of mefloquine-artemether compared with quinine on patients with complicated falciparum malaria. Transactions of The Royal Society of Tropical Medicine and Hygiene, 1988, 82, 665-666.

Sy N.D., Hoan D.B., Dung N.P., HuOng N.V., Binh L.N., SON M.V. and Meshnick S.R. : Treatment of malaria in Vietnam with oral artemisinin. American Journal of Tropical Medicine and Hygiene, 1993, 48, 398-402.

Warhurst D.C., Robinson B.L. and Peters W. : The blood schizontocidal action of erythromycin upon Plasmodium berghei. Annals of Tropical Medicine and Parasitology, 1976, 70, 253-258.

Warhust D.C., Robinson B.L. and Peters W. : The blood schizontocidal action of erythromycin against Plasmodium knowlesi infections in Macaca mulatta. Annals of Tropical Medicine and Parasitology, 1983, 77, 231-237.

Watt F., Loesuttivibool L., Shanks G.D., Doudreau E.F., Brown A.E., Pavanand K., Webster H.K. and Wechgritaya S. : Quinine with tetracycline for the treatment of drugresistant falciparum malaria in Thailand. American Journal of Tropical Medicine and Hygiene, 1992, 47, 108111.

White N. : Tetracycline for chloroquine-resistant malaria. Lancet, 1983, 1, 1271.

Win K., Than M. and Thwe Y. : Comparison of combinations of parenteral artemisinin derivatives plus oral mefloquine with intravenous quinine plus oral tetracycline for treating cerebral malaria. Bulletin of World Health Organization, 1992, 70, 777-782.

Ye Z., Li Z., Li G., Fu X., LiU H. and GAO M. : Effects of qinghaosu and chloroquine on the ultrastructure of the erythrocytic stage of $P$. falciparum in continuous cultivation in vitro. Journal of Traditional Chinese Medicine, 1983, 3, 95-102.

Ye Z., VAN Dyke K. and Castranova V. : The potentiating action of tetrandrine in combination with chloroquine or qinghaosu against chloroquine-sensitive and resistant falciparum malaria. Biochemical and Biophysical Research Communications, 1989, 165, 758-765.

Ye Z., VAN DYKe K. and WIMmer M. : Effect of artemisinin (qinghaosu) and chloroquine on drug-sensitive and drugresistant strains of Plasmodium falciparum malaria : Use of $[2,8-3 \mathrm{H}]$ adenosine as an alternative to $[\mathrm{G}-3 \mathrm{H}]$ hypoxanthine in the assessment of in vitro antimalarial activity. Experimental Parasitology, 1987, 64, 418-423.

Ye Z., VAN DYKE K. and YANG B. : Interaction of berbamine and chloroquine or artemisinin against chloroquine-sensitive and -resistant Plasmodium falciparum in vitro. Drug Development Research, 1993, 30, 229-237.

Accepté le 5 juillet 1994 